Published in final edited form as:

Exp Eye Res. 2020 September ; 198: 108140. doi:10.1016/j.exer.2020.108140.

\title{
Metabolic signature of eyelid basal cell carcinoma
}

\author{
Jiancheng Huang ${ }^{1,2,3,4}$, Jamie Schaefer ${ }^{3}$, Yekai Wang ${ }^{3,4}$, Lauren Gioia ${ }^{3}$, Ying Pei ${ }^{6}$, Xiaofei $^{2}$ \\ $\mathrm{Shi}^{6}$, Shanawar Waris ${ }^{3}$, Chen Zhao ${ }^{1,2}$, John Nguyen ${ }^{3,5,{ }^{*}, \text { Jianhai Du}}{ }^{3,4,{ }^{*}}$ \\ ${ }^{1}$ Eye Institute, Eye and ENT Hospital, Shanghai Medical College, Fudan University, Shanghai, \\ China, 200031
}

${ }^{2} \mathrm{NHC}$ Key Laboratory of Myopia (Fudan University), Key Laboratory of Myopia, Chinese Academy of Medical Sciences, and Shanghai Key Laboratory of Visual Impairment and Restoration (Fudan University), Shanghai, China, 200031

${ }^{3}$ Department of Ophthalmology, West Virginia University, Morgantown, WV 26506

${ }^{4}$ Department of Biochemistry, West Virginia University, Morgantown, WV 26506

${ }^{5}$ Department of Otolaryngology, West Virginia University, Morgantown, WV 26506

${ }^{6}$ Department of Industrial and Management System Engineering, West Virginia University, Morgantown, WV 26506

\section{Purpose}

Eyelid basal cell carcinoma (BCC) is the most common eyelid malignancy. Metabolic reprogramming is critical in tumorigenesis, but the metabolic feature of eyelid $\mathrm{BCC}$ remains elusive. In this study, we aim to reveal the metabolic profile in eyelid BCC using targeted metabolomics. Eyelid samples were collected from patients who had removal of BCC and from control patients who underwent blepharoplasty. Multivariate analysis of metabolomics data distinguished the two groups, indicating that eyelid $\mathrm{BCC}$ has significantly different metabolome than the healthy tissue. We found 16 increased and 11 decreased metabolites in the BCC tissues. These metabolites were highly enriched in the metabolism of nicotinamide adenine dinucleotide (NAD), glutathione metabolism, polyamine metabolism, and the metabolism of glycine, serine, threonine, arginine and proline. amino acid metabolism. Metabolites from NAD metabolism (Nicotinamide; Nicotinamide riboside; N1-Methylnicotinamide) had the highest sensitivity, specificity, and prediction accuracy in a prediction model for eyelid BCC. In conclusion, eyelid $\mathrm{BCC}$ has a signature change of cell metabolome. Metabolites in NAD metabolic pathways could potentially be biomarkers or therapeutic targets for eyelid BCC.

*Corresponding authors: John Nguyen, One Medical Center Dr, WVU Eye Institute, Morgantown, WV 26505; Phone: (304)-598-6944; Fax: (304)-598-6928; nguyenj@ wvumedicine.org., Jianhai Du, One Medical Center Dr, PO Box 9193, WVU Eye Institute, Morgantown, WV 26505; Phone: (304)-598-6903; Fax: (304)-598-6928; jianhai.du@ wvumedicine.org.

Publisher's Disclaimer: This is a PDF file of an unedited manuscript that has been accepted for publication. As a service to our customers we are providing this early version of the manuscript. The manuscript will undergo copyediting, typesetting, and review of the resulting proof before it is published in its final form. Please note that during the production process errors may be discovered which could affect the content, and all legal disclaimers that apply to the journal pertain. 


\section{Keywords}

Eyelid basal cell carcinoma; LC-MS/MS; Metabolism

\section{Introduction}

Eyelid malignancies represent approximately 5\% to $10 \%$ of all skin cancers (Tesluk, 1985). The incidence of eyelid malignancies is as high as 15.7 cases per 100,000 per year in the United States (Cook and Bartley, 1999). Basal cell carcinoma (BCC) is the most common eyelid malignancy, accounting for 85-95\% of all eyelid cancers (Silverman and Shinder, 2017; Yin et al., 2015). Usually located at the lower eyelid and medial canthal region, BCC rarely metastasizes, but it can locally invade into the orbit, sinuses, and brain to cause tissue destruction (Burgic et al., 2019). Although surgical resection with histologic control of margins is the preferred treatment for BCC (Margo and Waltz, 1993), radiation and cryotherapy are commonly used when surgery is not appropriate. However, they have higher recurrence rates than surgical resection (Fitzpatrick et al., 1984; Wong et al., 2002). The molecular mechanisms for the tumorigenesis are still unclear.

Metabolic reprograming is a hallmark of the tumor (Hanahan and Weinberg, 2011). To acquire sufficient nutrients such as glucose, amino acids, lipids, and nucleotide for proliferation, cancer cells biochemically adapted to alter their metabolic signaling pathways (Boroughs and DeBerardinis, 2015; Zhu and Thompson, 2019). Metabolic pathways such as polyamine metabolism and nicotinamide adenine dinucleotide (NAD) metabolism are involved in fundamental processes including transcription, DNA repair, cell cycle progression, apoptosis, and metabolism (Chiarugi et al., 2012; Damiani and Wallace, 2018). Many of these processes have been linked to cancer development (Hamanaka and Chandel, 2012; Parks et al., 2013). Ultraviolet (UV) radiation is the most crucial risk factor in the development of eyelid BCC (Yam and Kwok, 2014). UV can induce DNA damage to mutagenize genes in tumor promotion or suppression, driving tumor growth (Hussein, 2005). UV radiation could induce mutations in p53(Hussain et al., 2014), a tumor suppressor, by changing the dipyrimidine sites associated with methylated $\mathrm{CpG}$ ( $\mathrm{mCpG}$ ) to promote tumor growth (Ikehata and Ono, 2007). Interestingly, recent studies suggest that p53 suppresses tumors through metabolic regulations (Li et al., 2019; Liu et al., 2019). Poly (ADP-Ribose) Polymerase 1 (PARP-1) is a key enzyme in repairing the damaged DNA by UV, which relies on NAD, an essential metabolite in energy metabolism (Murata et al., 2019; Purohit et al., 2016). Additionally, a recent study of metabolites using NMR spectroscopy could discriminate cutaneous BCC from normal skin tissues (Mun et al., 2016). However, how eyelid BCC alters its metabolism in tumor pathogenesis remains unknown.

The metabolites are substrates and products in metabolic reactions, and the changes of metabolites directly reflect the status of cell metabolism. Targeted metabolomics using liquid chromatography tandem mass spectrometry (LC MS/MS) provides a sensitive, quantitative, and high throughput platform to measure metabolites in tumor tissues (Bondar et al., 2007; Mbeunkui et al., 2007; Yang et al., 2011). In this study, we aim to identify 
the metabolic signature of eyelid BCC by analyzing eyelid skin samples in patients with BCC using LC MS/MS. Among 83 targeted metabolites, we have identified 16 changed metabolites in NAD metabolism, glutathione metabolism, polyamine metabolism, and amino acid metabolism. Additionally, metabolites in NAD pathways have the highest sensitivity and specificity in a prediction model for eyelid BCC.

\section{Materials and Methods}

\subsection{Participants}

Study participants were patients who underwent blepharoplasty or basal cell carcinoma excision at the West Virginia University Eye Institute. The study was approved by the Institutional Review Board of West Virginia University and was in accordance with the Helsinki Declaration. Demographics including age, race, gender, diabetic status, body mass index (BMI), radiation history, and chemotherapy history, were summarized in Table 1.

\subsection{Sample collection}

The tissue specimens were obtained by one surgeon (JN). Normal skin tissues removed during upper eyelid blepharoplasty served as control samples. We chose the upper eyelid because of the availability of these patients. The basal carcinoma specimens were taken during lesion excision from either the upper or lower eyelid. The tissues were snap-frozen in liquid nitrogen and transferred to $-80^{\circ} \mathrm{C}$ for later analysis.

\subsection{Sample preparation}

All the samples were prepared and analyzed in the same batch. The tissues were quickly weighed and extracted for metabolites with cold $80 \%$ methanol in HPLC water (Vol//Vol) with a tissue homogenizer (Omni International) as reported (Yam et al., 2019). After precipitate proteins on dry ice for $30 \mathrm{~min}$, the supernatant was transferred to a fresh tube. The supernatant was then dried and reconstituted in $66 \%$ acetonitrile in HPLC water containing $10 \mathrm{mM}$ ammonium acetate (Vol/Vol). 13C-nicotinic acid (Toronto Research Chemicals) was added into each sample as the internal standard.

\subsection{Metabolite analysis for eyelid tissues}

LC-MS/MS was performed as previously reported (Huang et al., 2019). The extracts were analyzed by a Shimadzu LC Nexera X2 UHPLC coupled with a QTRAP 5500 LC MS/MS (AB Sciex). Chromatographic separation was performed by using an ACQUITY UPLC BEH Amide analytic column ( 2.1 X $50 \mathrm{~mm}, 1.7 \mu \mathrm{m}$, Waters) with a guard column and $0.2 \mu \mathrm{m}$ pre-column filter (Waters). The mobile phase was (A) water with $10 \mathrm{mM}$ ammonium acetate (pH 8.9) and (B) acetonitrile/water (95/5) with $10 \mathrm{mM}$ ammonium acetate (pH 8.2) (All solvents were LC-MS Optima grade from Fisher Scientific). The gradient elution is 95-61\% B in $6 \mathrm{~min}, 61-44 \% \mathrm{~B}$ at $8 \mathrm{~min}, 61-27 \% \mathrm{~B}$ at $8.2 \mathrm{~min}$, and $27-95 \% \mathrm{~B}$ at $9 \mathrm{~min}$. The column was equilibrated with $95 \% \mathrm{~B}$ at the end of each run. The source and collision gas were N2. We targeted 84 key metabolites in central carbon metabolism, and each metabolite was tuned for optimal transitions (Table S1). MultiQuant 3.0.2 software (AB Sciex) was applied to extract ions for peak area under the curve. All samples were analyzed in one batch.

Exp Eye Res. Author manuscript; available in PMC 2021 November 03. 


\subsection{Prediction model of NAD metabolic pathways}

We coded the response y as 1 or 0 representing samples with BCC or no BCC by MATLAB Software (MathWorks, MA, USA). The four metabolites in NAD metabolic pathways were represented as $\mathrm{x} 1, \mathrm{x} 2, \mathrm{x} 3, \mathrm{x} 4$. The prediction model was written as following:

$$
\begin{aligned}
& P\{y=1\}=\frac{e^{\beta_{0}+\beta_{1} x_{1}+\beta_{2} x_{2}+\beta_{3} x_{3}+\beta_{4} x_{4}}}{1+e^{\beta_{0}+\beta_{1} x_{1}+\beta_{2} x_{2}+\beta_{3} x_{3}+\beta_{4} x_{4}}} \\
& =\frac{e^{109.0-22.0 x_{1}+147.1 x_{2} \pm 851.3 x_{3}+32.8 x_{4}}}{1+e^{109.0-22.0 x_{1}+147.1 x_{2} \pm 851.3 x_{3}+32.8 x_{4}}}
\end{aligned}
$$

where $\mathrm{P}(\mathrm{y}=1)$ representing the probability of a certain patient with $\mathrm{BCC}$ tumor. To evaluate the accuracy of the predictive model, we performed k-fold cross validation.

\subsection{Data analysis}

Metabolomics data was analyzed with Partial least squares-discriminant analysis (PLSDA), variable importance in projection (VIP), heat map, volcano plot $(\mathrm{P}<0.05$ and fold change $>1.5$ ) and pathway analysis using MetaboAnalyst 4.0 (http://www.metaboanalyst.ca/) (Xia et al., 2012). The data was not transformed but normalized by a pool samples from the control group and then auto scaled before analysis with PLS-DA. Significantly changed metabolites were enriched for pathway analysis in the background of the human KEGG global metabolic database using MetaboAnalyst 4.0. A receiver operation characteristic (ROC) curve was generated using GraphPad Prizm (version 8.0; GraphPad Software, San Diego, CA, USA). PLS-DA model is used to transfer multivariant factors to univariant factor. Prediction model is created by MATLAB software (The MathWorks Inc., MA, USA). Data were presented as Mean \pm SEM unless otherwise stated. $\mathrm{P}<0.05$ was considered as statistical significance.

\section{Results}

\subsection{Clinical characteristics of participants}

The BCC participants consisted of 5 males and 3 females. The histopathologic subtypes of BCC were nodular $(n=5)$ and infiltrative/morpheaform $(n=3)$. Seventeen control participants included 10 males and 7 females without lesions who underwent blepharoplasty. Table 1 summarized the details of the clinical characteristics of these participants. There was no significant difference in genders, ages, BMI, and fasting blood glucose (BG) between the two groups (Table 1).

\subsection{Eyelid BCC alters metabolic profiles in tumor tissues}

We measured 84 metabolites in patients and normal tissues. To compare the overall difference, we used PLS-DA to analyze the multivariable dataset. PLS-DA analysis illustrated a significant separation between eyelid BCC and normal skin tissues in score plots, indicating cell metabolome in patient samples is significantly altered (Fig. 1A). To validate the PLS-DA model, we performed cross validation test. The R2 and Q2 were 0.92874 and 0.75515 respectively. 
indicating the high predictive relevance of the PLS-DA model (Table S2).To validate the PLS-DA model, we performed cross validation test. The R2 and Q2 values were 0.92874 and 0.75515 respectively (Table S2). The internal validation via permutation testing showed when the number of permutations were set at 2000, the accuracy was 0.9985, verifying the accuracy of our PLS-DA model (Fig S2)." Variable importance in projection (VIP) scores is associated with a significant difference in the PLS-DA. There were 21 metabolites with VIP scores set >1.2. Uracil, spermine, glucose 1-phosphate (G1P), erythritol, glycine, nicotinamide (NAM), N1-methylnicotinamide (N1-MNAM) were the top changed metabolites (VIP scores $>1.6$ ) (Fig. 1B). To differentiate the altered metabolites in BCC, we performed volcano plot analysis with a threshold of fold change $>1.5$ and $\mathrm{P}<$ 0.05 . Among the 84 metabolites, 27 metabolites were significantly changed in BCC samples, including 16 increased and 11 decreased metabolites (Fig. 1C, Table S3). Uracil, G1P, spermine, erythritol and glycine were top significantly changed metabolites; and kynurenine, carnosine, inosine monophosphate (IMP), nicotinamide riboside (NR) and ornithine had the biggest fold of changes in tissues with BCC. The top 25 changed metabolites from t-test were visualized in the heat map (Fig. 1D, Fig.S1, Table S2). To understand the metabolic pathways, we imported 27 changed metabolites into MetaboAnalyst 4.0 to identify the enriched pathways in the background of the Human sapiens KEGG pathway database. The top altered metabolic pathways included glutathione metabolism, NAD metabolism, Arginine and proline metabolism, Glycine, serine and threonine metabolism, Alanine, aspartate and glutamate metabolism, and Taurine and hypotaurine metabolism (Fig. 2). These findings in targeted metabolomics demonstrate that eyelid $\mathrm{BCC}$ has a distinctive metabolic signature from the normal skin tissue.

\subsection{Classification models}

NAD pathway had more changed metabolites (four in VIP score plots and three in $t$-test or volcano plots) than other affected metabolic pathways, and it was the top hit in both univariate and multivariate analysis (Fig. 1B and C). NR, a key precursor for salvage synthesis of NAD, decreased in BCC (Fig. 3B). NAM serves as both a precursor and a degradation product for NAD metabolism, and NAM could be methylated into N1-MNAM. Both NAM and N1-MNAM were accumulated in BCC tissues (Fig. 3C and D), indicating that there might be a high rate of NAD degradation in the BCC. As NAD metabolism is central in energy metabolism, and it is closely associated with other affected pathways such as glutathione and amino acid pathways, we performed further analysis of metabolites in NAD metabolism as predictors for BCC.

First, we analyzed these metabolites for Area under the curve - Receiver operating characteristic (AUC-ROC) curve for sensitivity and specificity as biomarkers. Higher AUC (maximum of AUC is 1) represents better sensitivity and specificity. Three of the four metabolites had higher AUC>0.8 (Nicotinamide: 0.978; N1-MNAM: 0.985; NR: 0.838) (Fig. 4A-D). To test whether NAD pathway is sufficient to discriminate BCC from the normal group, we performed multivariate modeling by applying the four metabolites for ROC analysis. The model generated from NAD metabolism pathway showed superior performance (AUC of 1) compared to any of the four metabolites (Fig. 4E). Prediction model based on these four metabolites from NAD metabolic pathway also exhibited high 
accuracy (Table 2). Taken together, we found NAD metabolic pathway could be potentially involved in BCC pathogenesis.

\section{Discussion}

In this study, we reveal the metabolic signature in eyelid BCC using targeted metabolomics with 27 significantly changed metabolites. Furthermore, NAD metabolism is impaired in BCC and its metabolites could potentially serve as biomarkers for BCC.

\subsection{BCC depletes Spermine and influences polyamine metabolism}

The changed metabolites are involved in the pathogenesis of eyelid BCC. In our study, we found significant change of spermine ( $\mathrm{p}<0.01$; AUC=1; FC $>2$; VIP $>1.2$ ) (Fig. 1B, Fig.S3, and Table S3). Spermidine and spermine are polyamines synthesized from the precursor putrescin, which is produced from ornithine in mammalian cells (Pegg, 2009). Polyamines are essential for cell proliferation, involving in the synthesis of protein and nucleotides (Casero et al., 2018). Rapidly growing tissues have higher levels of polyamine and growth-promoting hormonal stimuli enhance polyamine synthesis (Pegg et al., 1970; Russell and Snyder, 1968). Several oncogenic pathways including Myc, Ras, and PI3K regulate polyamine metabolism, and polyamine pathway, which has been proposed as a rational target for cancer treatment (Casero et al., 2018). Additionally, the activity of key enzyme (ornithine decarboxylase, ODC) in polyamine metabolism is increased in basal cell carcinoma (Coni et al., 2019). UV radiation, which is the most important factor in Eyelid BCC, activates ODC activity in mouse epidermis (Verma et al., 1979). Herein, we speculate that decreased spermine level might be caused by the enhanced consumption of the rapid-growing of tumor cells. Consistently, we found the precursors for spermine (arginine and glutamine) were increased.

\subsection{BCC and glucose metabolism}

Cancer cells are known for active glucose metabolism especially aerobic glycolysis (Vander Heiden et al., 2009). Glucose 6-phosphate (G6P) and G1P, key metabolites in glycolysis, gluconeogenesis and glycogenolysis, are increased in BCC. Hexokinase, the first ratelimiting enzyme in glycolysis, converts glucose into G6P while glucose 6 phosphatase, the final step in gluconeogenesis, hydrolyzes G6P to generate free glucose. Consistently, Hexokinase is critical for the initiation of multiple tumors (Han et al., 2019; Patra et al., 2013) and UV-irradiation induced DNA damage could suppress the expression of glucose 6 phosphatase (Kim et al., 2011). Furthermore, hexokinase II is tightly regulated by P53 (Mathupala et al., 1997), an important oncogene in Eyelid BCC. G1P is an important intermediates in glycogenlysis and it must be converted to G6P by the enzyme phosphoglucomutase (Bose and Le, 2018). The increase of G1P and G6P suggests that Eyelid BCC reprograms glucose metabolism into active catabolism for its growth.

\subsection{BCC alters NAD metabolism}

NAD is a pivotal cofactor for energy metabolism, DNA repair, and cell growth (Garten et al., 2009; Kennedy et al., 2016). NAD plays a central role in energy metabolism by carrying reducing equivalents to the electron transport chain to fuel oxidative phosphorylation (Canto 
et al., 2015). NAD serves as a substrate for PARP1 that plays an important role in DNA repair (Kim et al., 2005). PARP1 was shown to modify the effectiveness of the p53-mediated DNA damage response for different types of UV-exposure (Valenzuela et al., 2002). In mammalian cells, NAD is produced through either de novo synthesis by using tryptophan, or salvage synthesis by using nicotinic acid, NAM, Nicotinamide riboside (NR) and nicotinamide mononucleotide (NMN) (Revollo et al., 2004). Nicotinamide phosphoribosyl transferase (NAMPT) and nicotinamide mononucleotide adenylyl transferase (NMNAT) are two key enzymes that regulate NAD biosynthesis (Yaku et al., 2018). NAMPT converts NAM into NMN, which is the direct substrate of NMNAT to produce NAD. NNAMPT could be elevated in cancers to sustain rapid cellular proliferation and tumor survival (Galli et al., 2010). Nicotinamide riboside (NR) supplementation maintains the NAD levels preventing DNA damage (Tummala et al., 2014). In eyelid BCC, we speculate that DNA damage caused by UV radiation can activate PARP-1, which degrades NAD into NAM while activates NAD synthesis by reducing NR. The rise of NAM could be cleared by nicotinamide N-methyltransferase (NNMT) to be methylated into N1-methylnicotinamide (Johnson and Imai, 2018). NNMT is upregulated in many human cancers contributing to tumorigenesis (Ulanovskaya et al., 2013). N1-Methylnicotinamde is important in epigenetics by regulating protein and histone methylation (Pissios, 2017). The significant accumulation of N1-methylnicotinamide could alter the methylation landscape in BCC. Therefore, the dysregulated NAD metabolic pathway in BCC may be associated with tumor growth and DNA damage and repair.

A previous study identified 9 changed metabolites (alanine, aspartate, glycine, phosphocholine, acetate, creatine, fumarate, isoleucine, and lactate) in BCC by NMR spectroscopy (Mun et al., 2016). In our study, glycine and creatine were also altered, but other metabolites were unchanged. The difference could be due to the complexities of tumors and patient populations. The limitations of our study are the small sample size based on power analysis (Fig S4). Because of the nature of heterogeneity in tumors including histopathologic grade and subtypes, further studies with larger sample sizes and different histopathologic subtypes are required. Additionally, it will be important to trace these altered pathways in stable isotopes in human or animal models to elucidate the interaction of metabolic pathways.

\section{Conclusion}

Our findings reveal metabolic signature in eyelid BCC, pointing that BCC rewires cellular metabolome, especially NAD metabolism, polyamine metabolism, and glucose metabolism. This study should provide insights into the molecular pathogenesis of eyelid BCC and potential therapeutic benefits of targeting the altered metabolic pathways.

\section{Supplementary Material}

Refer to Web version on PubMed Central for supplementary material.

\section{Acknowledgment}

This work was supported by NIH Grants EY026030 (to J.D.) and Retina Research Foundation (to J.D.). 


\section{Reference}

Bondar OP, Barnidge DR, Klee EW, Davis BJ, Klee GG, 2007. LC-MS/MS quantification of Znalpha2 glycoprotein: a potential serum biomarker for prostate cancer. Clin Chem 53, 673-678. [PubMed: 17317883]

Boroughs LK, DeBerardinis RJ, 2015. Metabolic pathways promoting cancer cell survival and growth. Nat Cell Biol 17, 351-359. [PubMed: 25774832]

Bose S, Le A, 2018. Glucose Metabolism in Cancer. Adv Exp Med Biol 1063, 3-12. [PubMed: 29946772]

Burgic M, Iljazovic E, Vodencarevic AN, Burgic M, Rifatbegovic A, Mujkanovic A, Halilbasic M, Sinanovic M, 2019. Clinical Characteristics and Outcome of Malignant Eyelid Tumors: A Five-Year Retrospective Study. Med Arch 73, 209-212. [PubMed: 31402806]

Canto C, Menzies KJ, Auwerx J, 2015. NAD(+) Metabolism and the Control of Energy Homeostasis: A Balancing Act between Mitochondria and the Nucleus. Cell Metab 22, 31-53. [PubMed: 26118927]

Casero RA Jr., Murray Stewart T, Pegg AE, 2018. Polyamine metabolism and cancer: treatments, challenges and opportunities. Nat Rev Cancer 18, 681-695. [PubMed: 30181570]

Chiarugi A, Dolle C, Felici R, Ziegler M, 2012. The NAD metabolome--a key determinant of cancer cell biology. Nat Rev Cancer 12, 741-752. [PubMed: 23018234]

Coni S, Di Magno L, Serrao SM, Kanamori Y, Agostinelli E, Canettieri G, 2019. Polyamine Metabolism as a Therapeutic Target inHedgehog-Driven Basal Cell Carcinomaand Medulloblastoma. Cells 8.

Cook BE Jr., Bartley GB, 1999. Epidemiologic characteristics and clinical course of patients with malignant eyelid tumors in an incidence cohort in Olmsted County, Minnesota. Ophthalmology 106, 746-750. [PubMed: 10201597]

Damiani E, Wallace HM, 2018. Polyamines and Cancer. Methods Mol Biol 1694, 469-488. [PubMed: 29080189]

Fitzpatrick PJ, Thompson GA, Easterbrook WM, Gallie BL, Payne DG, 1984. Basal and squamous cell carcinoma of the eyelids and their treatment by radiotherapy. Int J Radiat Oncol Biol Phys 10, 449-454. [PubMed: 6725035]

Galli M, Van Gool F, Rongvaux A, Andris F, Leo O, 2010. The nicotinamide phosphoribosyltransferase: a molecular link between metabolism, inflammation, and cancer. Cancer Res 70, 8-11. [PubMed: 20028851]

Garten A, Petzold S, Korner A, Imai S, Kiess W, 2009. Nampt: linking NAD biology, metabolism and cancer. Trends Endocrinol Metab 20, 130-138. [PubMed: 19109034]

Hamanaka RB, Chandel NS, 2012. Targeting glucose metabolism for cancer therapy. J Exp Med 209, 211-215. [PubMed: 22330683]

Han CY, Patten DA, Lee SG, Parks RJ, Chan DW, Harper ME, Tsang BK, 2019. p53 Promotes chemoresponsiveness by regulating hexokinase II gene transcription and metabolic reprogramming in epithelial ovarian cancer. Mol Carcinog 58, 2161-2174. [PubMed: 31486135]

Hanahan D, Weinberg RA, 2011. Hallmarks of cancer: the next generation. Cell 144, 646-674. [PubMed: 21376230]

Huang J, Gu S, Chen M, Zhang SJ, Jiang Z, Chen X, Jiang C, Liu G, Radu RA, Sun X, Vollrath D, Du J, Yan B, Zhao C, 2019. Abnormal mTORC1 signaling leads to retinal pigment epithelium degeneration. Theranostics 9, 1170-1180. [PubMed: 30867823]

Hussain RM, Matthews JL, Dubovy SR, Thompson JM, Wang G, 2014. UV-independent p53 mutations in sebaceous carcinoma of the eyelid. Ophthalmic Plast Reconstr Surg 30, 392-395. [PubMed: 24777261]

Hussein MR, 2005. Ultraviolet radiation and skin cancer: molecular mechanisms. J Cutan Pathol 32, 191-205. [PubMed: 15701081]

Ikehata $\mathrm{H}$, Ono T, 2007. Significance of $\mathrm{CpG}$ methylation for solar UV-induced mutagenesis and carcinogenesis in skin. Photochem Photobiol 83, 196-204. [PubMed: 16620158]

Exp Eye Res. Author manuscript; available in PMC 2021 November 03. 
Johnson S, Imai SI, 2018. NAD (+) biosynthesis, aging, and disease. F1000Res 7, 132. [PubMed: 29744033]

Kennedy BE, Sharif T, Martell E, Dai C, Kim Y, Lee PW, Gujar SA, 2016. NAD(+) salvage pathway in cancer metabolism and therapy. Pharmacol Res 114, 274-283. [PubMed: 27816507]

Kim HJ, Jee HJ, Yun J, 2011. DNA damage induces down-regulation of PEPCK and G6P gene expression through degradation of PGC-1alpha. Acta Biochim Biophys Sin (Shanghai) 43, 589594. [PubMed: 21733854]

Kim MY, Zhang T, Kraus WL, 2005. Poly(ADP-ribosyl)ation by PARP-1: 'PAR-laying' NAD+ into a nuclear signal. Genes Dev 19, 1951-1967. [PubMed: 16140981]

Li L, Mao Y, Zhao L, Li L, Wu J, Zhao M, Du W, Yu L, Jiang P, 2019. p53 regulation of ammonia metabolism through urea cycle controls polyamine biosynthesis. Nature 567, 253-256. [PubMed: 30842655]

Liu J, Zhang C, Hu W, Feng Z, 2019. Tumor suppressor p53 and metabolism. J Mol Cell Biol 11, 284-292. [PubMed: 30500901]

Margo CE, Waltz K, 1993. Basal cell carcinoma of the eyelid and periocular skin. Surv Ophthalmol 38, 169-192. [PubMed: 8235999]

Mathupala SP, Heese C, Pedersen PL, 1997. Glucose catabolism in cancer cells. The type II hexokinase promoter contains functionally active response elements for the tumor suppressor p53. J Biol Chem 272, 22776-22780. [PubMed: 9278438]

Mbeunkui F, Metge BJ, Shevde LA, Pannell LK, 2007. Identification of differentially secreted biomarkers using LC-MS/MS in isogenic cell lines representing a progression of breast cancer. J Proteome Res 6, 2993-3002. [PubMed: 17608509]

Mun JH, Lee H, Yoon D, Kim BS, Kim MB, Kim S, 2016. Discrimination of Basal Cell Carcinoma from Normal Skin Tissue Using High-Resolution Magic Angle Spinning 1H NMR Spectroscopy. PLoS One 11, e0150328.

Murata MM, Kong X, Moncada E, Chen Y, Imamura H, Wang P, Berns MW, Yokomori K, Digman MA, 2019. NAD+ consumption by PARP1 in response to DNA damage triggers metabolic shift critical for damaged cell survival. Mol Biol Cell 30, 2584-2597. [PubMed: 31390283]

Parks SK, Chiche J, Pouyssegur J, 2013. Disrupting proton dynamics and energy metabolism for cancer therapy. Nat Rev Cancer 13, 611-623. [PubMed: 23969692]

Patra KC, Wang Q, Bhaskar PT, Miller L, Wang Z, Wheaton W, Chandel N, Laakso M, Muller WJ, Allen EL, Jha AK, Smolen GA, Clasquin MF, Robey B, Hay N, 2013. Hexokinase 2 is required for tumor initiation and maintenance and its systemic deletion is therapeutic in mouse models of cancer. Cancer Cell 24, 213-228. [PubMed: 23911236]

Pegg AE, 2009. Mammalian polyamine metabolism and function. IUBMB Life 61, 880-894. [PubMed: 19603518]

Pegg AE, Lockwood DH, Williams-Ashman HG, 1970. Concentrations of putrescine and polyamines and their enzymic synthesis during androgen-induced prostatic growth. Biochem J 117, 17-31. [PubMed: 5420953]

Pissios P, 2017. Nicotinamide N-Methyltransferase: More Than a Vitamin B3 Clearance Enzyme. Trends Endocrinol Metab 28, 340-353. [PubMed: 28291578]

Purohit NK, Robu M, Shah RG, Geacintov NE, Shah GM, 2016. Characterization of the interactions of PARP-1 with UV-damaged DNA in vivo and in vitro. Sci Rep 6, 19020. [PubMed: 26753915]

Revollo JR, Grimm AA, Imai S, 2004. The NAD biosynthesis pathway mediated by nicotinamide phosphoribosyltransferase regulates Sir2 activity in mammalian cells. J Biol Chem 279, 5075450763. [PubMed: 15381699]

Russell D, Snyder SH, 1968. Amine synthesis in rapidly growing tissues: ornithine decarboxylase activity in regenerating rat liver, chick embryo, and various tumors. Proc Natl Acad Sci U S A 60, 1420-1427. [PubMed: 4299947]

Silverman N, Shinder R, 2017. What's New in Eyelid Tumors. Asia Pac J Ophthalmol (Phila) 6, 143-152. [PubMed: 28399340]

Tesluk GC, 1985. Eyelid lesions: incidence and comparison of benign and malignant lesions. Ann Ophthalmol 17, 704-707. [PubMed: 4083660]

Exp Eye Res. Author manuscript; available in PMC 2021 November 03. 
Tummala KS, Gomes AL, Yilmaz M, Grana O, Bakiri L, Ruppen I, Ximenez-Embun P, Sheshappanavar V, Rodriguez-Justo M, Pisano DG, Wagner EF, Djouder N, 2014. Inhibition of de novo NAD(+) synthesis by oncogenic URI causes liver tumorigenesis through DNA damage. Cancer Cell 26, 826-839. [PubMed: 25453901]

Ulanovskaya OA, Zuhl AM, Cravatt BF, 2013. NNMT promotes epigenetic remodeling in cancer by creating a metabolic methylation sink. Nat Chem Biol 9, 300-306. [PubMed: 23455543]

Valenzuela MT, Guerrero R, Nunez MI, Ruiz De Almodovar JM, Sarker M, de Murcia G, Oliver FJ, 2002. PARP-1 modifies the effectiveness of p53-mediated DNA damage response. Oncogene 21 , 1108-1116. [PubMed: 11850828]

Vander Heiden MG, Cantley LC, Thompson CB, 2009. Understanding the Warburg effect: the metabolic requirements of cell proliferation. Science 324, 1029-1033. [PubMed: 19460998]

Verma AK, Lowe NJ, Boutwell RK, 1979. Induction of mouse epidermal ornithine decarboxylase activity and DNA synthesis by ultraviolet light. Cancer Res 39, 1035-1040. [PubMed: 427744]

Wong VA, Marshall JA, Whitehead KJ, Williamson RM, Sullivan TJ, 2002. Management of periocular basal cell carcinoma with modified en face frozen section controlled excision. Ophthalmic Plast Reconstr Surg 18, 430-435. [PubMed: 12439056]

Xia J, Mandal R, Sinelnikov IV, Broadhurst D, Wishart DS, 2012. MetaboAnalyst 2.0--a comprehensive server for metabolomic data analysis. Nucleic Acids Res 40, W127-133. [PubMed: 22553367]

Yaku K, Okabe K, Nakagawa T, 2018. NAD metabolism: Implications in aging and longevity. Ageing Res Rev 47, 1-17. [PubMed: 29883761]

Yam JC, Kwok AK, 2014. Ultraviolet light and ocular diseases. Int Ophthalmol 34, 383-400. [PubMed: 23722672]

Yam M, Engel AL, Wang Y, Zhu S, Hauer A, Zhang R, Lohner D, Huang J, Dinterman M, Zhao C, Chao JR, Du J, 2019. Proline mediates metabolic communication between retinal pigment epithelial cells and the retina. J Biol Chem 294, 10278-10289. [PubMed: 31110046]

Yang N, Feng S, Shedden K, Xie X, Liu Y, Rosser CJ, Lubman DM, Goodison S, 2011. Urinary glycoprotein biomarker discovery for bladder cancer detection using LC/MS-MS and label-free quantification. Clin Cancer Res 17, 3349-3359. [PubMed: 21459797]

Yin VT, Merritt HA, Sniegowski M, Esmaeli B, 2015. Eyelid and ocular surface carcinoma: diagnosis and management. Clin Dermatol 33, 159-169. [PubMed: 25704936]

Zhu J, Thompson CB, 2019. Metabolic regulation of cell growth and proliferation. Nat Rev Mol Cell Biol 20, 436-450. [PubMed: 30976106]

Exp Eye Res. Author manuscript; available in PMC 2021 November 03. 

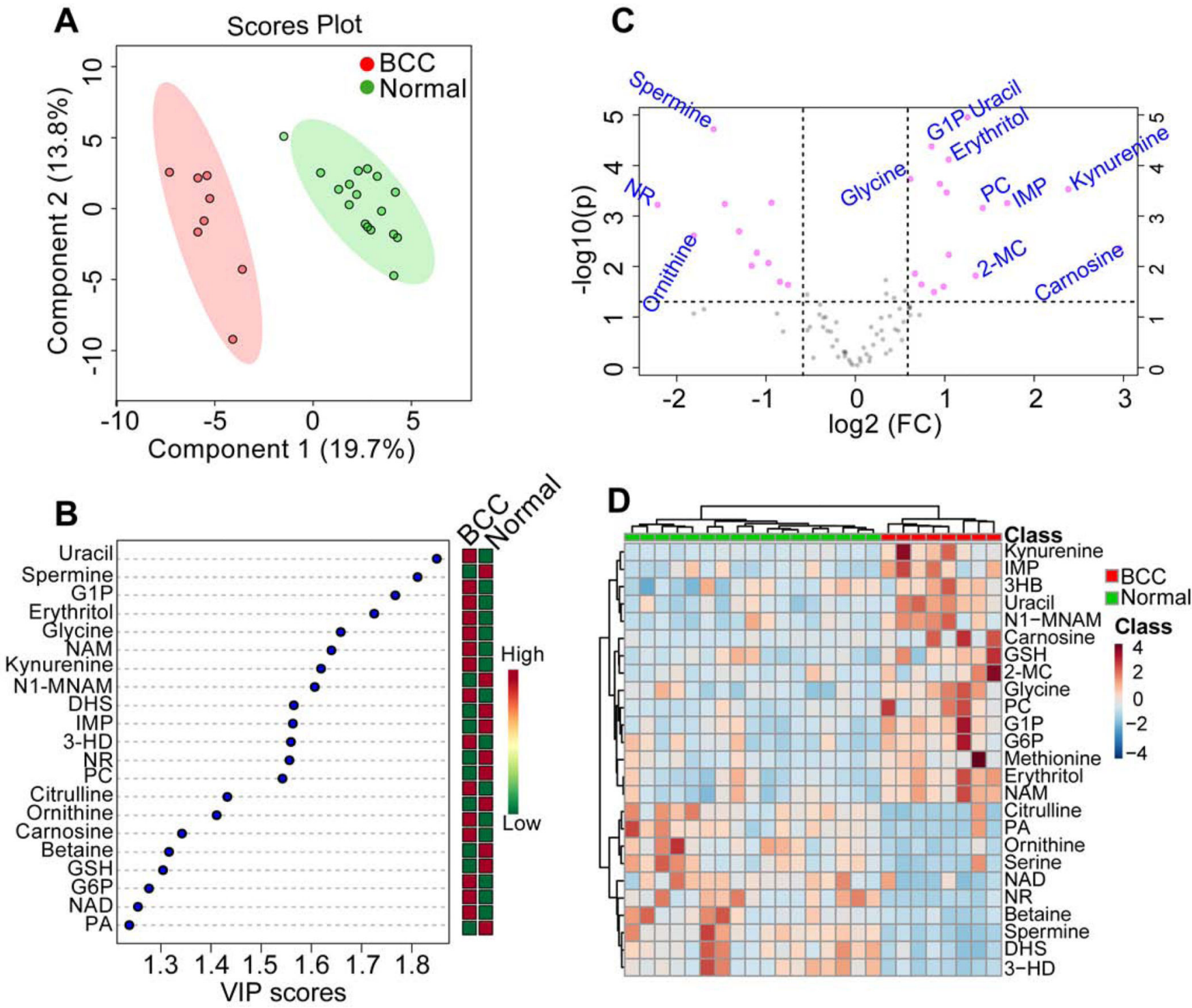

Figure 1: Overview of changed metabolites

(A) The PLS-DA score plot clearly discriminates BCC from normal subjects

(B) VIP score (VIP > 1.1) derived from PLS-DA model is shown.

(C) Volcano plot and column graph (Fold change $>2.0, P<0.05)$ of changed metabolites is shown.

(D) Heat map of top 25 changed metabolites.

NAM: nicotinamide; N1-MNAM: N1-Methylnicotinamide; 3-HD: 3-Hydroxykynurenine; Dihydrosphingosine: DHS; PC: Propionyl Carnitine; PA: Pyroglutamic Acid; 3-HB: 3-

Hydroxybutyrate; 2-MC: 2 Methylbutyryl Carnitine; 


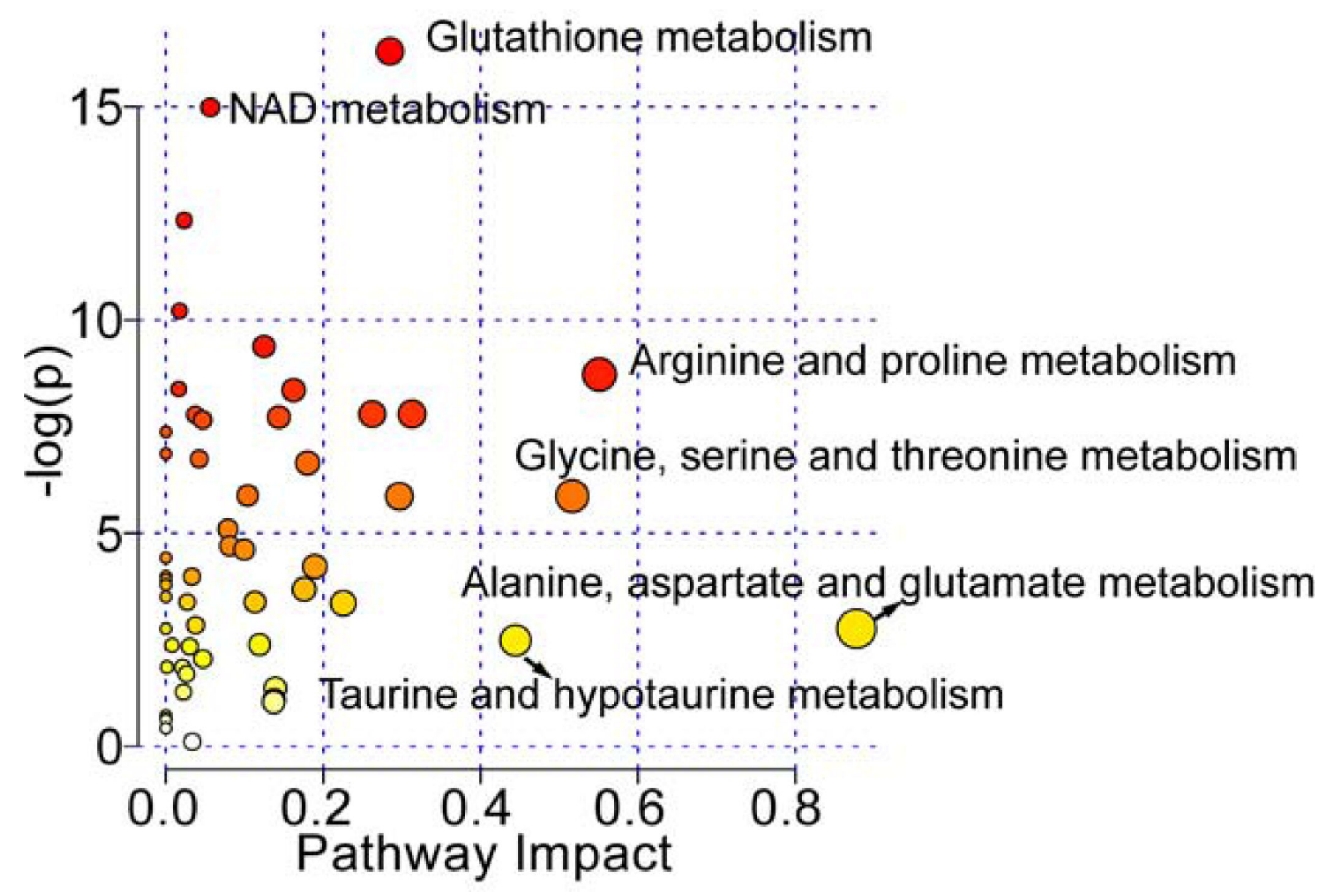

Figure 2:

Pathway analysis of changed metabolites 
A C
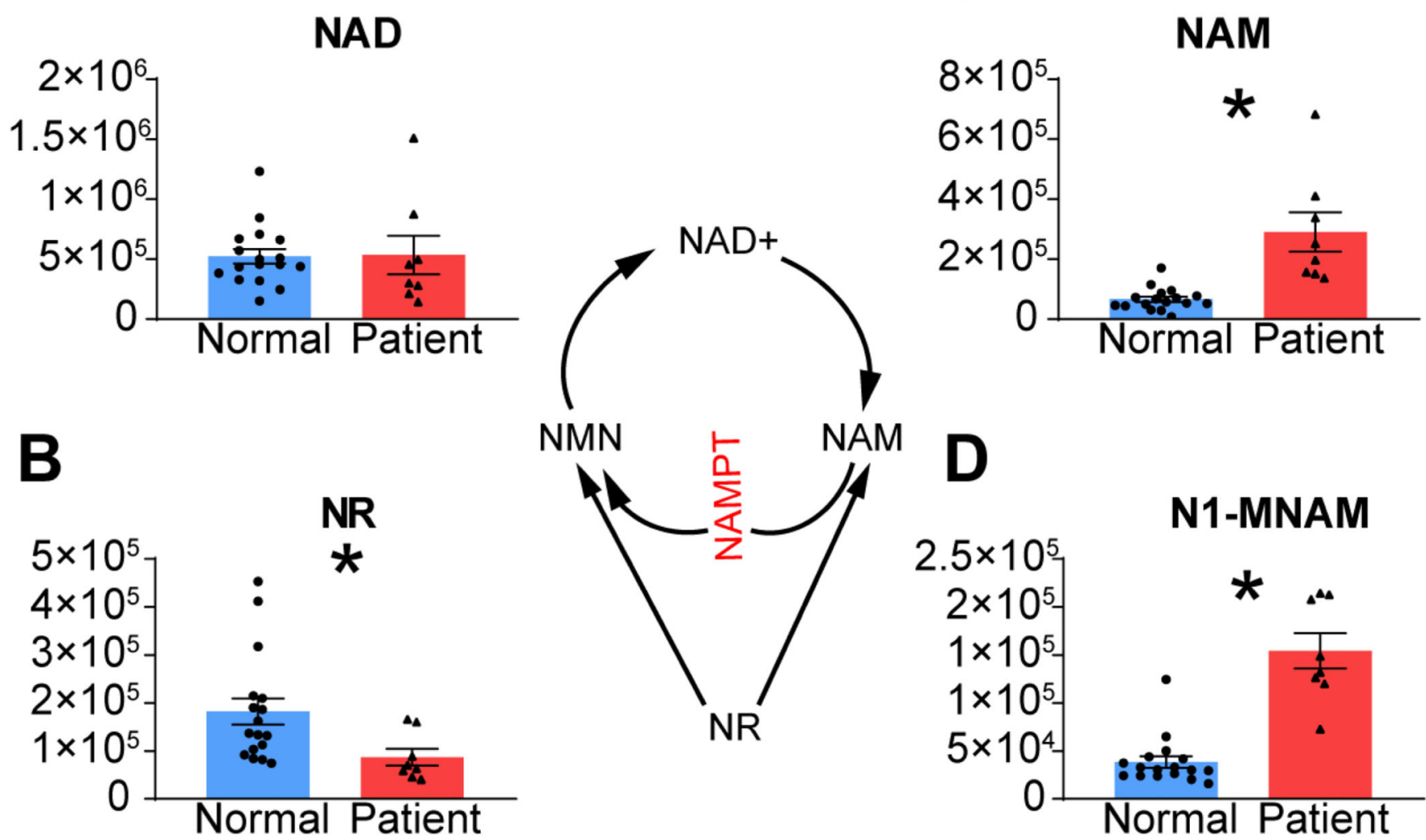

Figure 3: Univariate and multivariate analysis of metabolites from NAD metabolic pathway The change of metabolites from NAD metabolic pathway (* $P<0.05$ ) (NAD (A); NR (B); NAM (C); N1-MNAM (D)). 

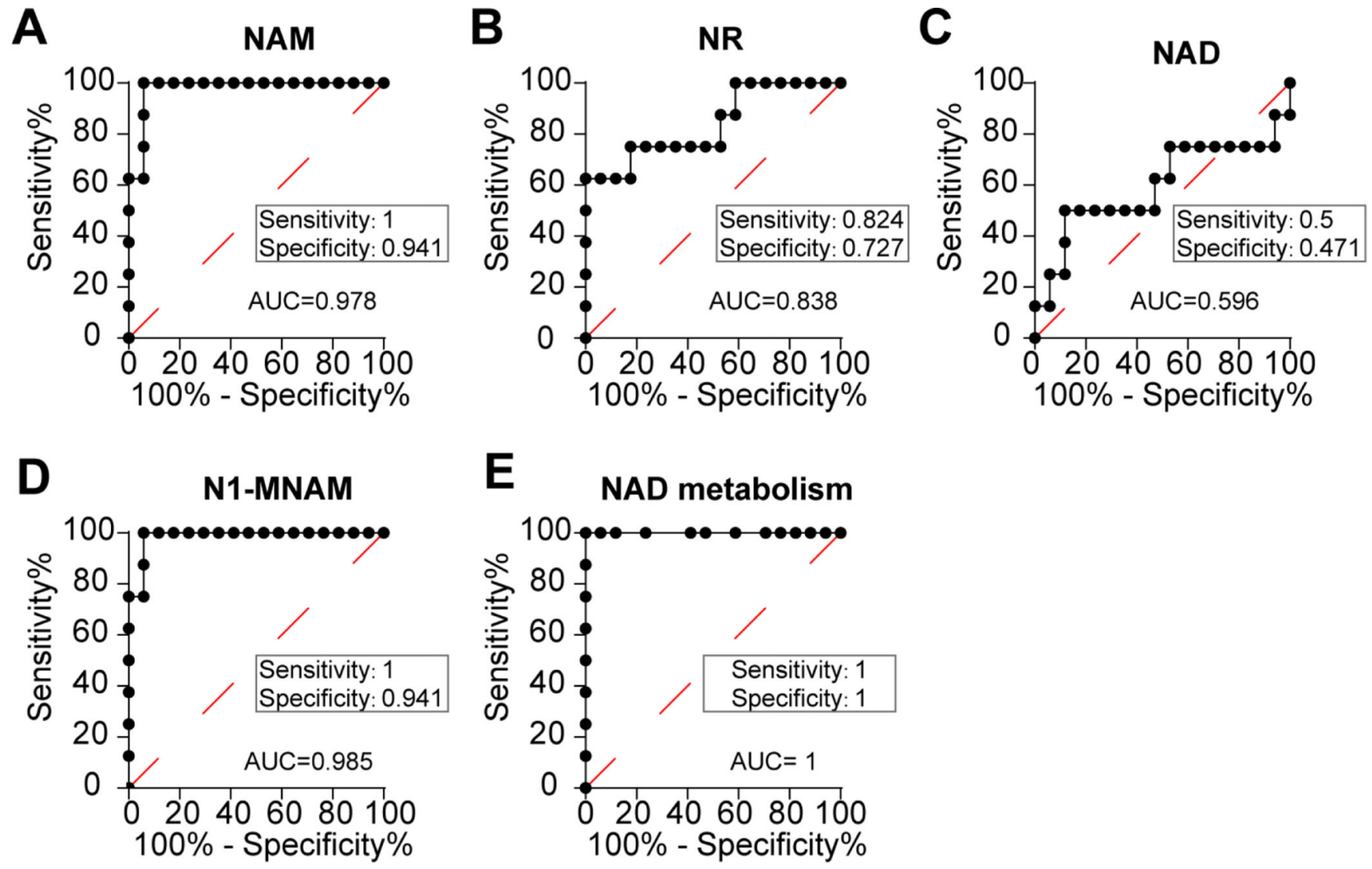

Figure 4: Multivariate analysis of NAD metabolic pathway

ROC curve of metabolites from NAD metabolic pathway (NAM (A); NR (B); NAD (C);

N1-MNAM (D)).

(E) ROC curve of NAD metabolic pathway (NAM; NR; NAD; N1-MNAM) in BCC and normal subjects after PLS-DA analysis

Using targeted metabolomics, we reveal metabolic features in the most common eyelid malignancy, basal cell carcinoma (BCC).

Eyelid BCC alters NAD metabolism, glutathione metabolism, polyamine metabolism, and amino acid metabolism.

Metabolites in NAD pathways are potential biomarkers and therapeutic targets for eyelid BCC. 
Table 1:

Clinical and Demographic information of human subjects included in this study

\begin{tabular}{lcc}
\hline Characteristics & Basal cell carcinoma $(\mathbf{N}=\mathbf{8})$ & Control $(\mathbf{N}=\mathbf{1 7})$ \\
\hline Ages (years; Mean \pm SD) & $65.875 \pm 19.915$ & $71.235 \pm 10.27$ \\
Gender & 5 & 10 \\
Male & 3 & 7 \\
Female & & \\
Race/Ethnicity & $8 / 8$ & $17 / 17$ \\
White & $28.288 \pm 7.637$ & $32.421 \pm 4.729$ \\
BMI (Kg/m2) & $100.75 \pm 30.208$ & $100.471 \pm 33.509$ \\
Fasting BG (mg/dl) & $0 / 8$ & $0 / 17$ \\
Radiation history & $0 / 8$ & $0 / 17$ \\
Chemotherapy history & & \\
Histology & 5 & \\
Nodular & 3 & \\
Infiltrative/morpheaform & & \\
\hline
\end{tabular}

Exp Eye Res. Author manuscript; available in PMC 2021 November 03. 
Table 2:

7-cross validation results for the prediction model based on NAD metabolic pathway

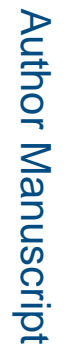

\begin{tabular}{|l|c|}
\hline & Prediction accuracy \\
\hline Fold 1 & $100 \%$ \\
\hline Fold 2 & $100 \%$ \\
\hline Fold 3 & $75 \%$ \\
\hline Fold 4 & $100 \%$ \\
\hline Fold 5 & $100 \%$ \\
\hline Fold 6 & $100 \%$ \\
\hline Fold 7 & $75 \%$ \\
\hline
\end{tabular}

